\title{
Receptor Activity-Modifying Protein 2 (RAMP2) alters glucagon receptor trafficking in hepatocytes with functional effects on receptor signalling
}

\author{
Emma Rose McGlone ${ }^{1}$, Yusman Manchanda ${ }^{2}$, Ben Jones ${ }^{1}$, Phil Pickford ${ }^{1}$, Asuka Inoue ${ }^{3}$, David Carling ${ }^{4}$, \\ Stephen R. Bloom ${ }^{1}$, Tricia Tan ${ }^{1, *, 5,7}$, Alejandra Tomas ${ }^{2, *, 5,6}$
}

\section{ABSTRACT}

Objectives: Receptor Activity-Modifying Protein 2 (RAMP2) is a chaperone protein which allosterically binds to and interacts with the glucagon receptor (GCGR). The aims of this study were to investigate the effects of RAMP2 on GCGR trafficking and signalling in the liver, where glucagon (GCG) is important for carbohydrate and lipid metabolism.

Methods: Subcellular localisation of GCGR in the presence and absence of RAMP2 was investigated using confocal microscopy, trafficking and radioligand binding assays in human embryonic kidney (HEK293T) and human hepatoma (Huh7) cells. Mouse embryonic fibroblasts (MEFs) lacking the Wiskott-Aldrich Syndrome protein and scar homologue (WASH) complex and the trafficking inhibitor monensin were used to investigate the effect of halted recycling of internalised proteins on GCGR subcellular localisation and signalling in the absence of RAMP2. NanoBiT complementation and cyclic AMP assays were used to study the functional effect of RAMP2 on the recruitment and activation of GCGR signalling mediators. Response to hepatic RAMP2 upregulation in lean and obese adult mice using a bespoke adeno-associated viral vector was also studied.

Results: GCGR is predominantly localised at the plasma membrane in the absence of RAMP2 and exhibits remarkably slow internalisation in response to agonist stimulation. Rapid intracellular accumulation of GCG-stimulated GCGR in cells lacking the WASH complex or in the presence of monensin indicates that activated GCGR undergoes continuous cycles of internalisation and recycling, despite apparent GCGR plasma membrane localisation up to 40 min post-stimulation. Co-expression of RAMP2 induces GCGR internalisation both basally and in response to agonist stimulation. The intracellular retention of GCGR in the presence of RAMP2 confers a bias away from $\beta$-arrestin-2 recruitment coupled with increased activation of $G_{\alpha s}$ proteins at endosomes. This is associated with increased short-term efficacy for glucagon-stimulated cAMP production, although long-term signalling is dampened by increased receptor lysosomal targeting for degradation. Despite these signalling effects, only a minor disturbance of carbohydrate metabolism was observed in mice with upregulated hepatic RAMP2.

Conclusions: By retaining GCGR intracellularly, RAMP2 alters the spatiotemporal pattern of GCGR signalling. Further exploration of the effects of RAMP2 on GCGR in vivo is warranted.

(C) 2021 The Authors. Published by Elsevier GmbH. This is an open access article under the CC BY license (http://creativecommons.org/licenses/by/4.0/).

Keywords Glucagon receptor; Receptor activity-modifying protein 2; G protein-coupled receptors; Endocytic trafficking; Intracellular signalling; Carbohydrate metabolism

\section{INTRODUCTION}

Glucagon acts through the glucagon receptor (GCGR), a prototypical G protein-coupled receptor (GPCR) of the secretin-like (class B) family [1]. The effects of glucagon (GCG) on the liver include increased hepatic glucose output by stimulation of glycogenolysis and gluconeogenesis, inhibition of de novo lipogenesis and increased fatty acid oxidation [2-4]. Type 2 diabetes mellitus (T2DM) and nonalcoholic fatty liver disease (NAFLD) are characterised by high GCG levels and GCG resistance [5,6]; manipulation of GCG signalling is a potential pharmacological strategy for the treatment of these conditions $[7,8]$. Intracellular trafficking of other GPCRs from the secretin-

\footnotetext{
${ }^{1}$ Section of Endocrinology and Investigative Medicine, Department of Metabolism, Digestion and Reproduction, Imperial College London, London, UK ${ }^{2}$ Section of Cell Biology and Functional Genomics, Department of Metabolism, Digestion and Reproduction, Imperial College London, London, UK ${ }^{3}$ Graduate School of Pharmaceutical Sciences, Tohoku University, Sendai, Japan ${ }^{4}$ MRC London Institute of Medical Sciences, Imperial College London, London, UK

5 Joint senior and corresponding authors.

6 Alejandra Tomas: Section of Cell Biology and Functional Genomics, Room 329, 3rd floor ICTEM Building, Hammersmith Campus, Du Cane Road, London, W12 0NN, UK.

7 Tricia Tan: Section of Endocrinology and Investigative Medicine, 6th Floor Commonwealth Building, Imperial College London, Du Cane Road, London, W12 OHS, UK.

*Corresponding author. E-mails: t.tan@imperial.ac.uk (T. Tan), a.tomas-catala@imperial.ac.uk (A. Tomas).
}

Received May 9, 2021 • Revision received July 1, 2021 • Accepted July 9, 2021 • Available online 13 July 2021

https://doi.org/10.1016/j.molmet.2021.101296 
like family has been demonstrated to play a key role in the regulation of receptor signalling outputs [9-13], suggesting that modifying intracellular trafficking may be a tractable approach to modulate GCGR signalling.

Receptor activity-modifying proteins (RAMPs) are mammalian accessory proteins that interact allosterically with the vast majority of GPCRs $[14,15]$. Their actions are wide-reaching and highly variable, but include modulation of receptor trafficking, changes to ligand specificity and alteration of intracellular responses to receptor activation [16]. As RAMPs interact with their cognate GPCRs in a complex lipid membrane environment, their effects on receptor function vary depending on the cell type in which they are studied [17]. RAMP2 (but not RAMP1 or RAMP3) co-localises with the GCGR and alters its pharmacology in certain cell types [18-20]. We have observed a reduction in cell surface GCGR in the presence of RAMP2 [19]. The aims of the present study were twofold: first, to analyse the effects of RAMP2 on the intracellular trafficking and spatiotemporal regulation of GCGR signalling in more detail; and second, to investigate the effects of the interaction between RAMP2 and GCGR in hepatocytes, a physiologically relevant cell type.

\section{MATERIALS AND METHODS}

Please also see Supplementary Methods.

\subsection{Peptides}

All peptides were purchased from Insight Biotechnology. Glucagon (129) (GCG) and glucagon-like peptide 1 (GLP-1) $(7-36) \mathrm{NH}_{2}$, the predominant bioactive forms of GCG and GLP-1, respectively, were used for all experiments, except where fluorescent glucagon and GLP-1 peptide conjugates featuring a fluorescein isothiocyanate (FITC-GCG and FITC-GLP-1) were used to monitor ligand binding and/or uptake. FITC-GLP-1 has been previously described and validated [21]. For radioligand binding assays, GCG was directly iodinated in-house $\left(1^{125}\right.$ from Hartmann Analytic) and purified with reversed-phase high performance liquid chromatography [22].

\subsection{Cell lines}

HEK293T, MEF (flox/flox and WASH-out, a gift from Professor Daniel Billadeau, Mayo Clinic, Rochester, USA) and Huh7 hepatoma cells were maintained in DMEM supplemented with $10 \%$ FBS and $1 \%$ penicillin/ streptomycin and cultured at $37{ }^{\circ} \mathrm{C}$ in a $5 \% \mathrm{CO}_{2}$ atmosphere. INS-1 832/3 cells (a gift from Professor Christopher Newgard, Duke University Medical Center, Durham, USA) were maintained in RPMI supplemented with $11 \mathrm{mM}$ glucose, 10\% FBS, $10 \mathrm{mM}$ HEPES, $2 \mathrm{mM}$ Lglutamine, $1 \mathrm{mM}$ pyruvate, $50 \mu \mathrm{M} \beta$-mercaptoethanol and $1 \%$ penicillin/streptomycin. A stable clone of Huh7 cells expressing human GCGR (Huh7-GCGR) was generated from a previously-described multiclonal cell population [19] by flow cytometric sorting of cells labelled with FITC-GCG, and subsequently maintained in DMEM, 10\% FBS, 1\% penicillin/streptomycin and $1 \mathrm{mg} / \mathrm{mL}$ G418 (Thermo Fisher).

\subsection{Transfections}

Transient transfections of SNAP-GCGR, SNAP-GLP-1R (both Cisbio), RAMP2, GCGR-GFP (both Origene), RAMP2-CFP, empty vector (EV)CFP (both GeneCopoeia), Nb37-GFP (a gift from Professor Roshanak Irannejad, University of California San Francisco, USA), CLIP-RAMP2 [cloned in-house and sequence-verified from RAMP2 (Origene) and CLIP- $\beta 2$-AR (a gift from Professor Davide Calebiro, University of Birmingham)], TGN-marker (Venus-tagged GRIP domain, made in-house), GLP-1R-GFP (a gift from Professor Alessandro Bisello, University of
Pittsburgh, USA), HALO-GCGR and HALO-GLP-1R (both made inhouse), Rab5-Venus (a gift from Professor Kevin Pfleger, University of Western Australia) and plasmids for the NanoBiT complementation assays (see Supplementary Methods) were performed using Lipofectamine 2000 (Thermo Fisher) for HEK293T and Huh7 cells or by electroporation with the Neon transfection system (Thermo Fisher) for MEF cells, according to the manufacturer's instructions. Experiments were performed 24 hours after transfection unless otherwise indicated. Reverse transfection with siRNA against RAMP2 or a Silencer Select negative control (both Ambion), again with Lipofectamine 2000, was used to downregulate RAMP2. Reagents were added at the time of plating the cells, and experiments performed 72 hours later.

\subsection{Antibodies}

SNAP-GCGR was detected with an anti-SNAP-tag rabbit polyclonal antibody (P9310S, New England Biolabs, 1/500) followed by goat antirabbit IgG H\&L HRP (ab6271, Abcam, 1/2,000). Post-stripping, tubulin was labelled with anti- $\alpha$-tubulin mouse monoclonal antibody (T5168, Sigma, 1/5,000) followed by sheep anti-mouse secondary antibody HRP (ab6721, Abcam, 1/5,000). For liver samples, the following antibodies were used: anti-RAMP2 sc-365240 at 1/500 dilution, secondary sc-516102 at 1/1,000 dilution (both from Santa Cruz Biotechnology); and anti-GAPDH mab374 at 1/500 dilution (Merck), secondary \#15014 at 1/10,000 (Active Motif).

\subsection{Animal care}

Experiments were performed in accordance with the UK Animals (Scientific Procedures) Act 1986 and approved by the Animal Welfare and Ethical Review Board at Imperial College London. C57BL/6J male mice (Charles River) were group housed in cages at a controlled temperature $\left(22^{\circ} \mathrm{C}\right)$, with a 12-hour light-dark cycle and free access to water. All interventions were performed during the light cycle. Mice were weaned and maintained on standard chow $(11 \% \mathrm{kcal}$ from fat and $62 \%$ from carbohydrate, SDS Rm3).

\subsection{Up-regulation of hepatic RAMP2 in mice}

Mouse Ramp2 and GFP (control) under the albumin promoter were constructed in an AAV2/8 pseudotyped adeno-associated virus vector (Vector BioLabs). At age 6 weeks, mice were administered a tail vein intravenous injection of $1 \times 10^{11}$ gene count of AAV-alb-GFP or AAValb-Ramp2. Mice were randomised for injections and returned to their original cages; that is, mice with hepatic RAMP2 up-regulation were co-housed with control mice. After 3 weeks, metabolic tests on lean mice were performed. At age 13 weeks, mice were transferred to a high-fat diet containing 60\% kcal from fat (Research Diets D12492). After a further 8 weeks, metabolic tests were performed on obese mice. All tests were performed on 5-hour fasted mice unless otherwise specified. Tail vein blood glucose was measured using a handheld glucometer (Nexus, GlucoRx) before and at indicated intervals after intraperitoneal injections of glucose ( $2 \mathrm{mg} / \mathrm{kg}$ body weight), insulin $(0.5$ or $1 \mathrm{U} / \mathrm{kg}$ of Actrapid human insulin for lean and obese mice, respectively; Novo Nordisk), pyruvate (2 g/kg, Sigma) or GCG (10 nmol/ $\mathrm{kg}$ body weight). Obese mice were dosed with glucose and GCG according to estimated lean weight of the same strain, sex and age of mouse maintained on standard chow $(31 \mathrm{~g})$. After the study period, mice were culled via decapitation following a 5-hour period of food restriction. The liver was harvested rapidly and snap frozen in liquid nitrogen. Hepatocytes were isolated using a collagenase perfusion, as previously described [23]. After washing and plating, they were serumstarved overnight before cyclic adenosine monophosphate (cAMP) assays were performed. 


\subsection{Data and statistical analyses}

All statistical analysis was performed using GraphPad Prism 9.0. For cAMP and NanoBiT complementation assays, $E_{\max }$ and $\log _{5} \mathrm{C}_{50}$ were derived for each repeat and then compared using paired t-tests. Manders' coefficient was calculated by comparing the confocal images in Fiji using the Coloc2 plugin to illustrate the extent of co-localisation between two fluorophore markers [24]. Signal bias calculations were derived from NanoBiT data. Baseline-corrected curves, normalised to vehicle, were generated for GCG-stimulated LgBiT-mini- $\mathrm{G}_{\mathrm{s}}$, LgBiTmini- $G_{q}$ and $L g B i T-\beta$-arrestin-2 recruitment data to GCGR-SmBiT in both RAMP2- and pcDNA3.1-transfected HEK293T cells and used to calculate the area under the curve (AUC) over 30 minutes (see Supplementary Methods). RAMP2/pcDNA3.1 AUC ratios were subsequently calculated for each recruited factor and compared for statistical significance using a one-way ANOVA with Dunnett's post-hoc test. All other specific statistical tests are indicated in figure legends. AUC was calculated from $y=0$. Statistical significance was considered at $\mathrm{p}<0.05$.

\section{RESULTS}

\subsection{GCGR exhibits rapid internalisation and recycling to the cell membrane upon agonist stimulation}

Unlike other glucagon-like peptide receptors, GCGR does not appear to exhibit short-term agonist-stimulated internalisation [12,25>,26]. To investigate this phenomenon, we used a fluorescent ligand (FITC-GCG) to stimulate SNAP-GCGR-expressing HEK293T cells, which were chosen because they do not express endogenous RAMPs [20]. FITCGCG has comparable potency for CAMP production to glucagon at the GCGR $\left(\log \mathrm{EC}_{50}-8.8\right.$ vs $-9.1 ; p=0.11$, Supplementary Figure $\left.1 \mathrm{~A}\right)$. Although FITC-GCG rapidly accumulated inside the cell after only a few minutes of stimulation, internalisation of the GCGR occurred much more slowly (Figure 1A,B). This is in stark contrast to the rapid internalisation exhibited by both FITC-GLP-1 and the glucagon-like peptide 1 receptor (GLP-1R) (Figure 1C,D), in agreement with previous observations indicating that, following agonist stimulation, the GLP-1R is rapidly internalised within 10-15 minutes of GLP-1 exposure $[11,12,25]$. Whereas SNAP-GLP-1R tightly co-localised with its ligand throughout the course of the experiment, SNAP-GCGR no longer co-localised with FITC-GCG after 10 minutes of agonist stimulation (Figure 1E). Similar findings of minimal GCGR internalisation in contrast to substantial GLP-1R internalisation were observed in a pancreatic beta cell line after 30 minutes of agonist stimulation (Supplementary Figure 1B,C), and in HEK293T cells using receptors with a C-terminal GFP tag and unlabelled agonists (Supplementary Figure 1D,E). Given the discrepancy between internalisation of GCGR and its ligand, we hypothesised that the apparent lack of GCGR internalisation is illusory: for shorter stimulation periods, the GCGR would internalise along with its ligand, which it would deposit intracellularly, then would rapidly recycle to the cell membrane, further undergoing rapid cycles of internalisation and recycling before a substantial proportion of the receptor would accumulate intracellularly. To investigate this hypothesis, we used mouse embryonic fibroblasts (MEFs) in which the Wiskott-Aldrich syndrome protein and scar homologue (WASH) complex has been knocked out [27]. The WASH complex is an important regulator of vesicle recycling in many cell types, deficiency of which non-specifically traps internalised GPCRs in endosomal compartments [27-30]. Following a short period of GCG stimulation, we found the GCGR was retained intracellularly in MEFs lacking the WASH complex (WASH-out MEFs), while the receptor was localised primarily at the plasma membrane in control MEF flox/flox cells (Figure 1F,G). To corroborate this, we also used the ionophore monensin to block endocytic recycling [31] in HEK293T cells expressing SNAP-GCGR during stimulation with GCG (Supplementary Figure $1 F, G$ ). In the presence of monensin, we observed increased intracellular accumulation of GCG-stimulated GCGR. This effect was reversed after a 1-hour monensin washout. Taken together, these results indicate that in the absence of RAMP2, GCGR undergoes a continuous cycle of internalisation followed by intracellular ligand deposition and plasma membrane recycling, which leads to a slow course of intracellular GCGR accumulation.

\subsection{GCGR accumulates intracellularly in the presence of RAMP2} In other contexts, RAMPs have been demonstrated to influence postendocytic receptor trafficking [32,33]. To investigate whether RAMP2 affects the subcellular localisation of GCGR, we co-transfected HEK293T cells with SNAP-GCGR and CLIP-RAMP2 (a RAMP2 derivative with a short N-terminal CLIP-tag) and labelled the tagged proteins with fluorescently-conjugated membrane-impermeable surface SNAPtag and CLIP-tag probes. In the absence of RAMP2, SNAP-GCGR was observed predominately at the cell membrane; in cells expressing RAMP2, however, a proportion of SNAP-GCGR was visible inside the cell where it colocalised with CLIP-RAMP2 (Manders' coefficient 0.45) (Figure 2A). Similar results were obtained with HALO-tagged GCGR (Supplementary Figure 2A). Co-transfection with Rab5-Venus, an endosomal marker, indicated that SNAP-GCGR and CLIP-RAMP2 colocalise in early endosomes following agonist stimulation (Figure 2B). Given the physiological relevance of hepatocytes for GCG signalling and the cell line dependence of RAMP activity, we next examined this phenomenon in Huh7 hepatoma cells, which express low levels of endogenous RAMP2 [19]. Huh7 cells were similarly transfected with SNAP-GCGR, with or without untagged RAMP2 co-expression, and surface GCGR labelled with a fluorescent surface SNAP-tag probe. Less SNAP-GCGR was observed at the cell surface with exogenous RAMP2 (Figure $2 C, D ; p=0.03$ ). To further investigate this phenomenon using an untagged GCGR, we performed a radioligand binding assay to measure GCGR cell surface density in Huh7 cells stably expressing GCGR (Huh7-GCGR) at $4{ }^{\circ} \mathrm{C}$ (to inhibit receptor endocytosis) where RAMP2 was either up- or down-regulated. We observed a reduced apparent GCGR cell surface density where RAMP2 was upregulated (Figure 2E,F), while surface GCGR was increased where RAMP2 was down-regulated (Figure 2G; $p=0.0096$ ).

We repeated the assay using FITC-GCG at $4{ }^{\circ} \mathrm{C}$ to quantify the level of surface GCGR in Huh7-GCGR cells with and without RAMP2 coexpression under basal conditions and after GCG stimulation. We found that the presence of RAMP2 decreased plasma membrane GCGR under both conditions (Figure 2H,I). Additionally, we measured an increase in the internalisation propensity of the GCGR in the presence of RAMP2 in HEK293T cells with both the SNAP-tagged (Figure 2J) and the HAL0-tagged GCGR (Supplementary Figure 2A,B). Taken together, these findings demonstrate that the presence of RAMP2 increases the intracellular localisation of GCGR, either by increasing the rate of GCGR endocytosis or by reducing the rate of GCGR recycling to the plasma membrane, in both basal and GCG-stimulated conditions, a consistent finding across different cell lines.

\subsection{Up-regulation of RAMP2 increases cAMP production acutely and engenders signalling bias in hepatoma cells}

Agonist-stimulation of GCGR leads to recruitment of $\mathrm{G} \alpha_{s}, G \alpha_{i}$ and $G \alpha_{q}$ proteins, triggering intracellular events that lead to modulation of cAMP levels, as well as recruitment of the $\beta$-arrestins (Supplementary Figure $3 A, B)$. It has been previously demonstrated that GCG-stimulated cAMP 
A

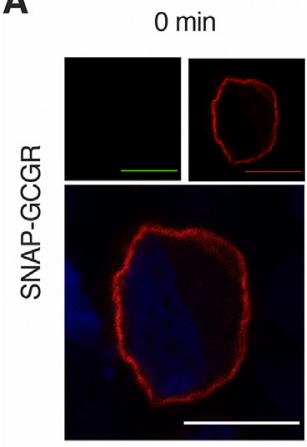

C

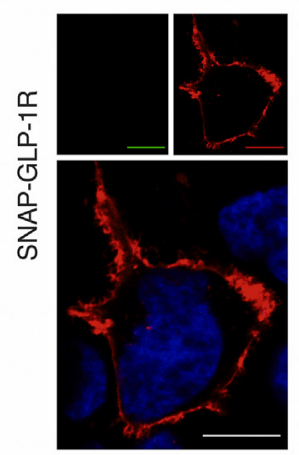

D

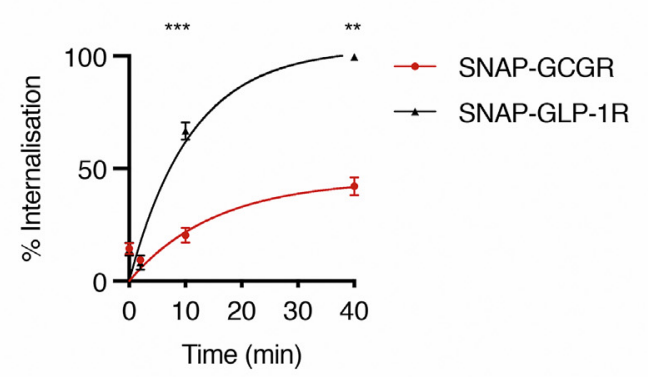

F

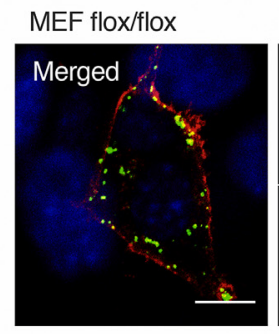

$2 \min$

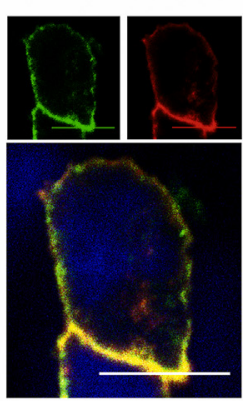

$2 \min$
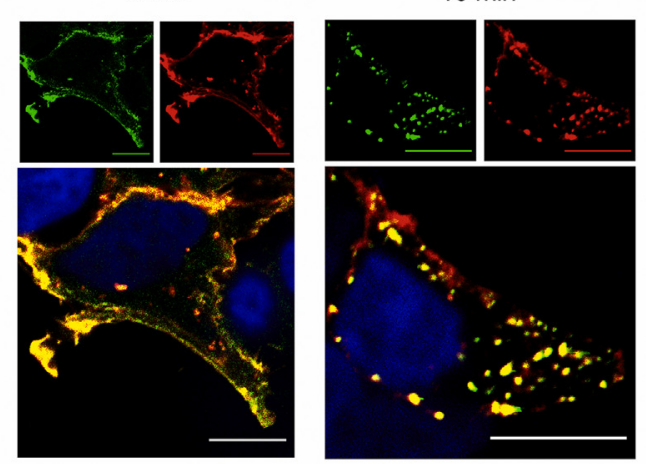

B
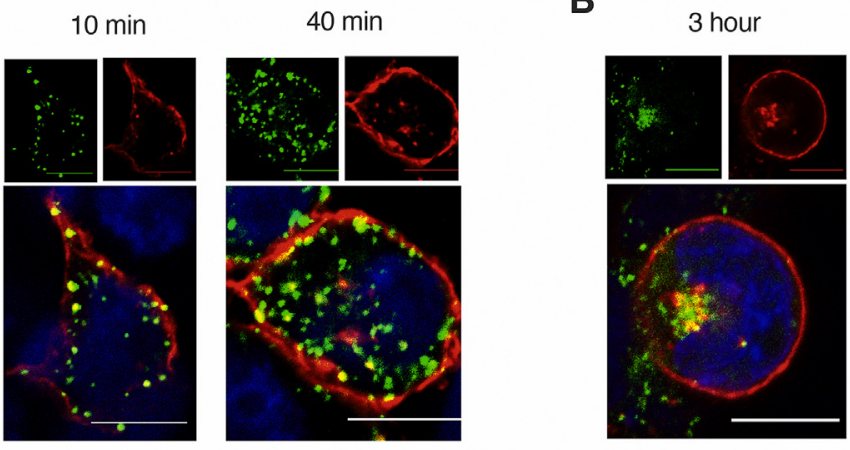

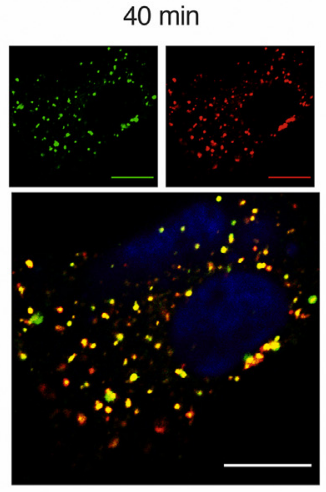

E

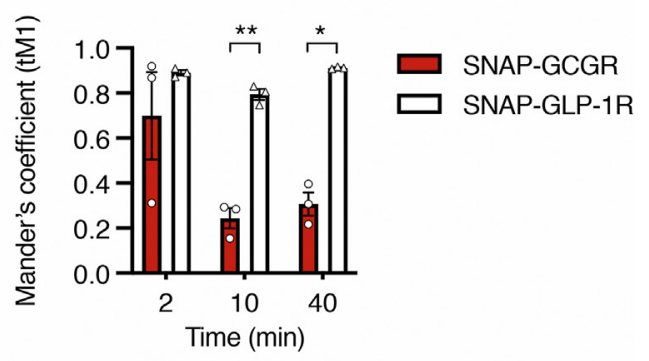

G

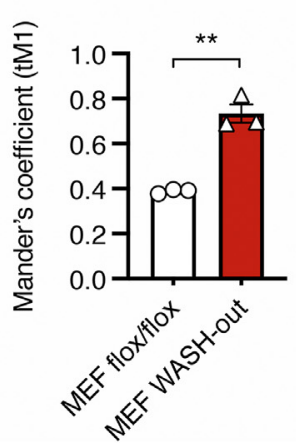

Figure 1: GCGR recycles to the plasma membrane following GCG stimulation. A-C: HEK293T cells transfected with SNAP-GCGR (A, B) or SNAP-GLP-1R (C), labelled with SNAP-Surface 549 (red), and stimulated with 100 nM FITC-GCG or FITC-GLP-1 (green), respectively, for the indicated time periods; nuclei stained with DAPI (blue); scale bars $=10 \mu \mathrm{m}$. D: Percentage of internalisation of SNAP-GCGR vs. SNAP-GLP-1R in response to their respective ligands, mean \pm SEM of $n=4$ pooled data shown, fitted to exponential plateau. E: Quantification of degree of co-localisation (Manders' coefficient) of the two receptors with their respective ligands at the indicated time-points as illustrated in A and C. F: MEF cells with or without WASH knockout (MEF flox/flox control or WASH-out), transfected with SNAP-GCGR (labelled with SNAP-Surface 549, red) and stimulated with FITC-GCG (green) for $30 \mathrm{~min}$; nuclei stained with DAPI (blue); scale bars $=10 \mu \mathrm{m}$. G: Quantification of degree of co-localisation of SNAP-GCGR and FITC-GCG in each MEF cell subtype as illustrated in F. Statistical significance was analysed using 2-way ANOVA with Sidak's multiple comparison test ( $\mathrm{D}$ and $\mathrm{E})$ and unpaired $\mathrm{t}$-test (G); ${ }^{*} \mathrm{p}<0.05$; ${ }^{* *} \mathrm{p}<0.01, * * * \mathrm{p}<0.001$. 
A

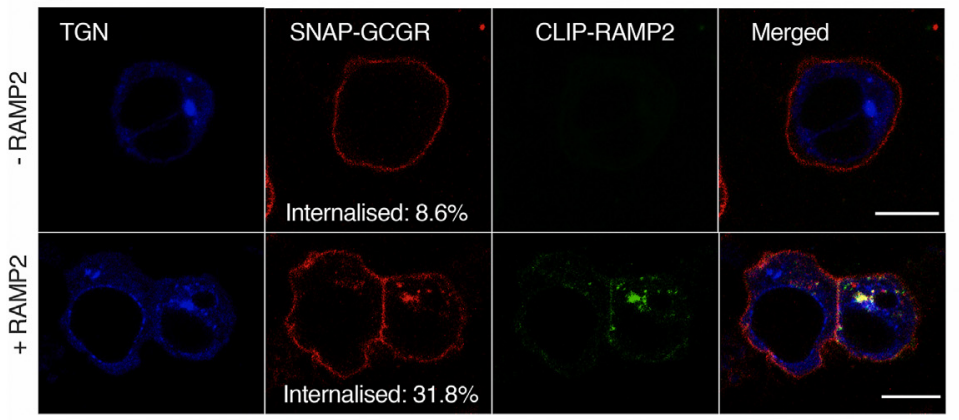

C

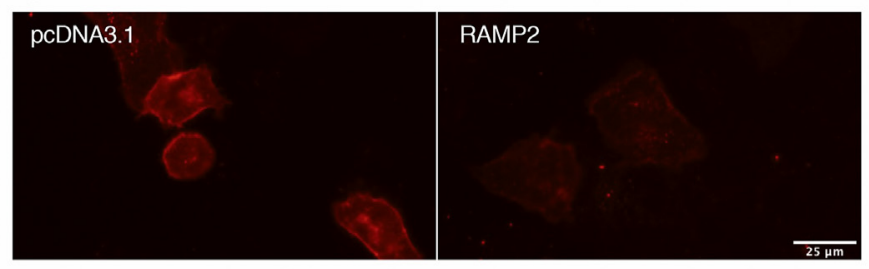

E
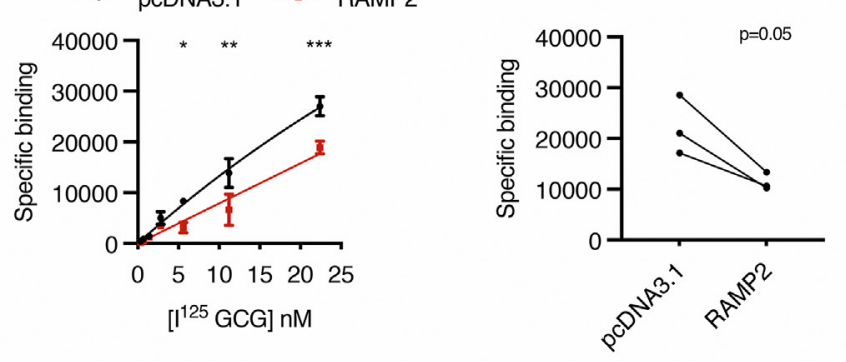

B
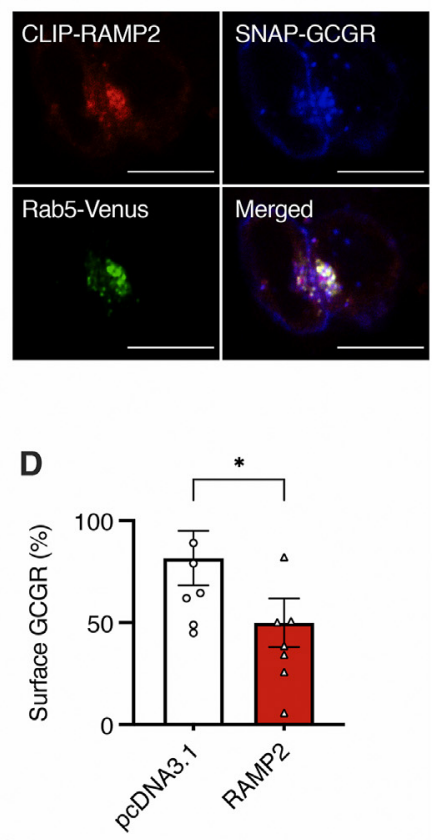

G

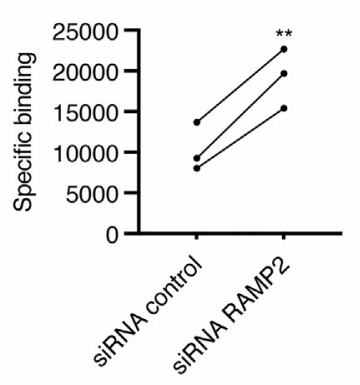

H

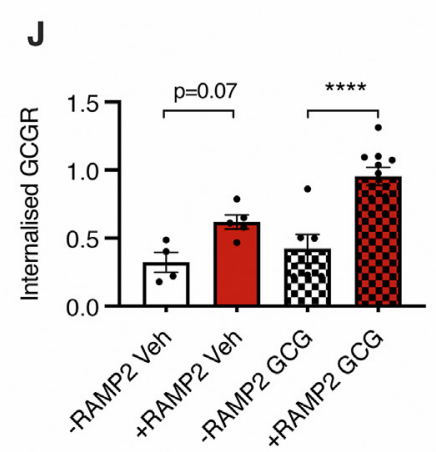

Figure 2: RAMP2 accelerates internalisation of GCGR. A: HEK293T cells transfected with a trans-Golgi network (TGN) marker (blue), SNAP-GCGR (labelled with SNAP-Surface 649 , red) and with or without CLIP-RAMP2 (labelled with CLIP-Surface 547, green); scale bars $=10 \mu \mathrm{m}$. B: HEK293T cells transfected with Rab5-Venus (green), SNAP-GCGR (labelled with SNAP-Surface 649, blue) and CLIP-RAMP2 (labelled with CLIP-Surface 547, red) and then treated with $100 \mathrm{nM}$ GCG for 40 min; scale bars = $10 \mu \mathrm{m}$. C: Huh7 cells co-transfected with SNAP-GCGR (labelled with SNAP-Surface 649, red) and CLIP-RAMP2 or control empty vector (pcDNA3.1); scale bars $=25 \mu \mathrm{m}$. D: Surface SNAP-GCGR density in unstimulated Huh7 cells from $C ;{ }^{*} p<0.05$. E-G: Specific binding of $\mathrm{I}^{125}$-GCG to Huh7-GCGR: increasing $\mathrm{I}^{125}$-GCG concentrations in control (pcDNA3.1) vs. RAMP2 overexpression (E), and at a single concentration of $5.6 \mathrm{nM} \mathrm{I}{ }^{125}-\mathrm{GCG}$ in control (pcDNA3.1) vs. RAMP2 up-regulation (F), or control vs. RAMP2 silencing (G), measured in counts over $240 \mathrm{~s}$; data are mean \pm SEM of $n=3-4$ experiments, normalised to total protein levels; ${ }^{*} p<0.05 ;{ }^{* *} p<0.01 ; * * * p<0.001$. H: Huh7-GCGR cells transfected with RAMP2 and then treated with either vehicle or $100 \mathrm{nM} \mathrm{GCG}$ for $30 \mathrm{~min}$ at $37^{\circ} \mathrm{C}$ prior to addition of FITC-GCG (green) at $4{ }^{\circ} \mathrm{C}$; nuclei stained with DAPI (blue); scale bars $=10 \mu \mathrm{m}$. I: Quantification of surface GCGR by FITC-GCG binding in cells from H; ${ }^{*} \mathrm{p}<0.05$; J: HEK293T cells transfected with SNAP-GCGR and either empty vector (EV)-CFP (-RAMP2) or RAMP2-CFP (+RAMP2) and then stimulated with vehicle or $100 \mathrm{nM} \mathrm{GCG,} \mathrm{SNAP-GCGR} \mathrm{seen} \mathrm{inside} \mathrm{the} \mathrm{cell} \mathrm{expressed} \mathrm{as} \mathrm{ratio} \mathrm{of} \mathrm{total} \mathrm{cellular} \mathrm{GCGR;} \mathrm{***p}<0.0001$. Data are mean \pm SEM of at least $n=3$ independent experiments. Statistical significance was analysed using paired t-tests (D-I) and one-way ANOVA with Sidak's post-hoc test (J). 
A Huh: 30 minutes GCG

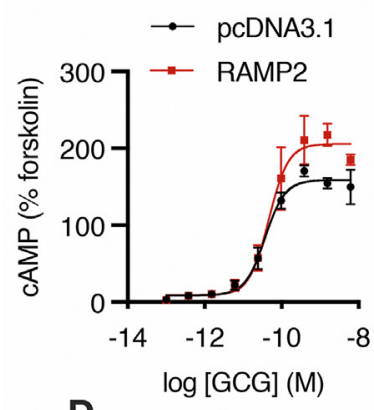

D Mini-Gi recruitment

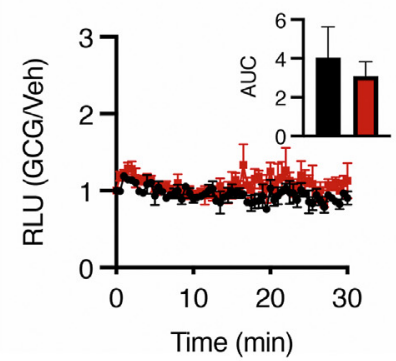

G

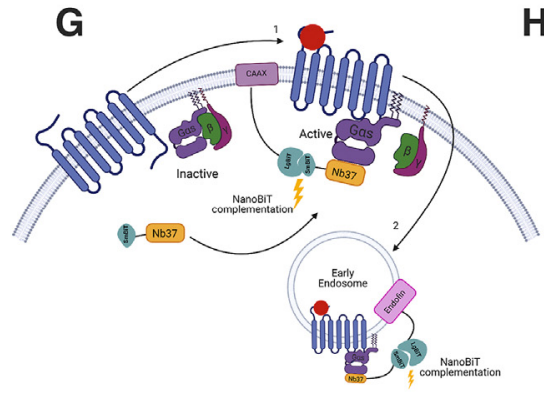

J

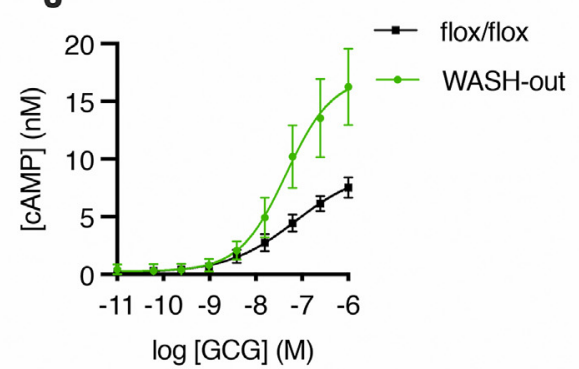

$\mathbf{L}$

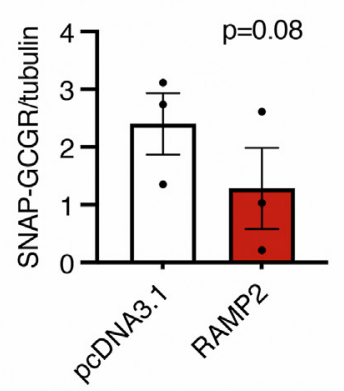

B

E

H
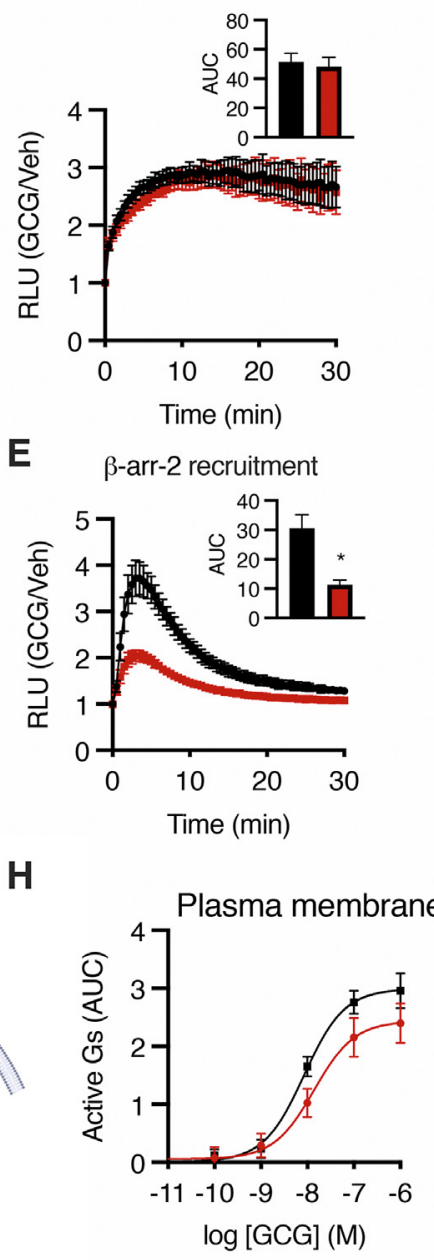

K

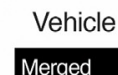

$$
\text { Merged }
$$
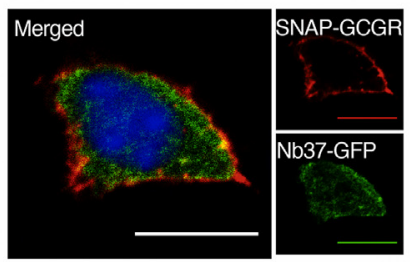

- RAMP2

+ RAMP2

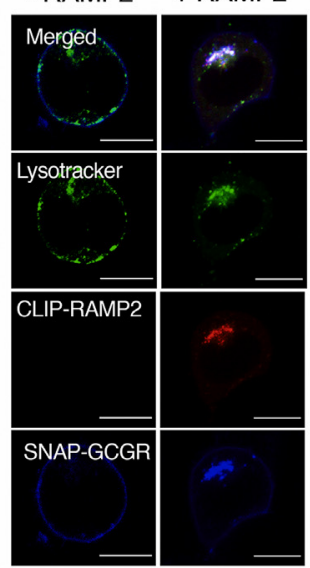

C Mini-Gq recruitment

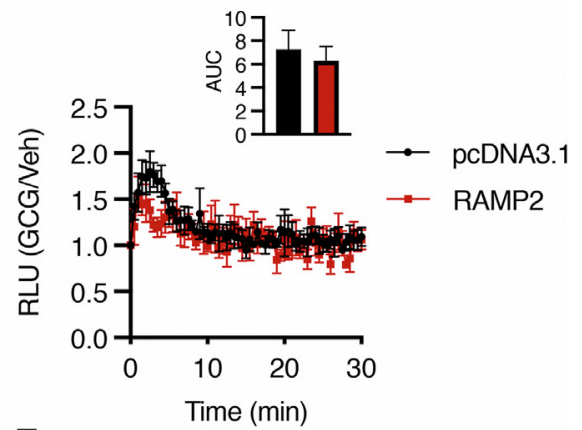

$\mathbf{F}$

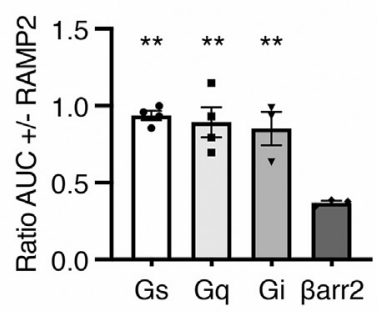

I

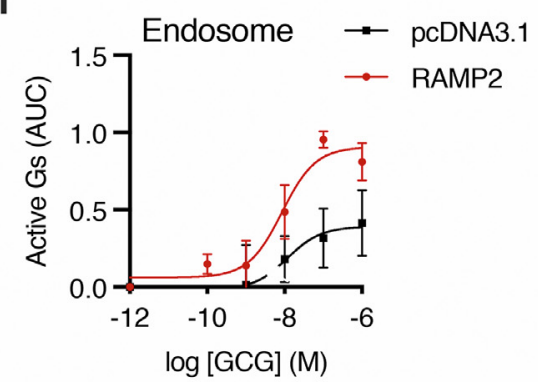

GCG

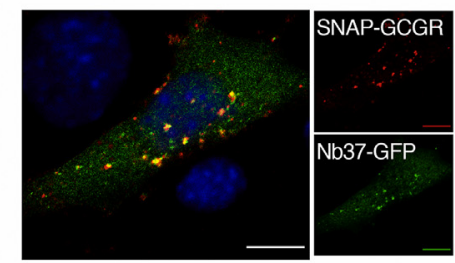

$\mathbf{N}$

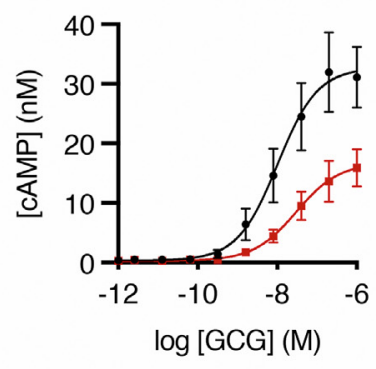


production is increased in the presence of RAMP2 in HEK293T [20] and Chinese hamster ovary (CHO) cells [19]. Here, we determine that in Huh7-GCGR hepatoma cells, the upregulation of RAMP2 also acutely increases efficacy for cAMP production in response to GCG stimulation (Figure $3 A$; Table 1); this is consistent with our previous finding that a reduction of RAMP2 in Huh7 cells is associated with a decrease in cAMP production with GCG [19]. One possible mechanism explaining this finding could be differential recruitment of intracellular mediators to the GCGR in the presence of RAMP2; indeed, previous work indicated that RAMP2 reduces recruitment of the inhibitory $G$ protein subunit $G \alpha_{i}$ to GCGR in yeast strains expressing $G$ protein chimeras [20]. To further explore the effect of RAMP2 on the recruitment of $G$ proteins and $\beta$-arrestins to the GCGR, we employed a NanoBiT complementation assay in HEK293T cells, which we have previously used to assess transducer coupling by the GCG family of receptors $[25,34]$. We observed a reduction in $\beta$-arrestin-2 recruitment to the GCGR in the presence of RAMP2, with no difference in recruitment of mini-G $\alpha_{s},-G \alpha_{i}$ and $-G \alpha_{q}$ proteins (Figure $3 B-E$ ), leading to a bias away from $\beta$-arrestin-2 recruitment when compared to the recruitment of all three mini-G subunits when RAMP2 is upregulated (Figure 3F).

\subsection{Endosomal retention of GCGR increases agonist-stimulated cAMP production}

Increased intracellular retention of GCGR in the presence of RAMP2 could potentiate CAMP accumulation, as sustained signalling from endosomes has been observed in several related secretin-family GPCRs and may be linked to the formation of GPCR-G protein megacomplexes [29-32]. To investigate this hypothesis, we designed a novel NanoBiT subcellular $\mathrm{G}$ protein activation assay to distinguish agonist-stimulated activation of the GCGR at the plasma versus endosomal membranes. Here, we co-express a plasma membrane (CAAX) or endosomal (Endofin) marker fused to the large BiT (LgBiT) subunit of the Nanoluc luciferase together with nanobody-37 (Nb37), a single domain antibody which binds specifically to nucleotide-free $\mathrm{G} \alpha_{\mathrm{S}}$ in complex with active receptors [35], fused to a complementary small subunit (SmBiT) of Nanoluc. Under this configuration, when the two nanoluciferase subunits are closely apposed, a quantitative luminescent signal is generated [36], indicating the presence of active $\mathrm{G} \alpha_{\mathrm{S}}$ in endosomal or plasma membrane compartments (see Figure $3 G$ for a schematic of the assay). In HEK293T cells in the absence of GCG stimulation, we observed a trend towards diminished basal levels of activation at the plasma membrane in the presence of RAMP2 $(p=0.08)$ but not at the endosomal compartment (Supplementary Figure 3C,D), in keeping with our previous finding of reduced surface GCGR levels in basal conditions. Following baseline normalisation, we observed no difference in the activation of plasma membrane GCGR upon GCG stimulation in the presence of RAMP2 (Figure 3H; Table 1), but recorded a significant increase in both efficacy and potency for endosomal GCGR signalling with RAMP2 (Figure 3l; Table 1). Furthermore, by artificially inducing GCGR intracellular retention in the MEFs devoid of the WASH complex (Figure 1D), we similarly increased the efficacy for cAMP production after GCG stimulation (Figure 3J, Table 1). Moreover, upon glucagon stimulation in these MEF WASH-out cells, we could observe co-localisation within intracellular puncta of a GFP fusion of Nb37 and the SNAP-GCGR, indicating that the GCGR is active and signalling at this intracellular location (Figure $3 \mathrm{~K}$ ). Because a proportion of intracellular GPCRs can be targeted from endosomes towards the degradative pathway [37], we next investigated whether the propensity for degradation of GCGR might be increased by RAMP2 co-expression. We observed that prolonged (4hour) GCG stimulation in the presence of RAMP2 resulted in a trend towards reduced total SNAP-GCGR levels $(p=0.08$; Figure $3 \mathrm{~L}$ and Supplementary figure 3E). This was associated with greater colocalisation of SNAP-GCGR with low $\mathrm{pH}$ endosomal compartments marked by LysoTracker in the presence of RAMP2 after 3 hours of GCG stimulation (Figure 3M). We also investigated how cAMP accumulation would be affected by RAMP2 co-expression in Huh7-GCGR cells in the context of prolonged GCG stimulation and found that, although RAMP2 was associated with increased efficacy for CAMP generation when measured acutely (Figure $3 A$ ), this effect was reversed after 24 hours of GCG exposure (Figure 3N and Table 1).

\subsection{Hepatic RAMP2 upregulation does not grossly affect carbohydrate metabolism in lean or obese adult male mice} To investigate whether there was a biological effect of the observed changes in GCG-stimulated cAMP accumulation in hepatocytes following upregulation of RAMP2, we used an adeno-associated virus vector to upregulate the murine Ramp2 gene under the control of the albumin promoter (AAV-alb-RAMP2) in hepatocytes of adult male mice. RAMP2 protein in the livers of treated mice was increased 3-fold (1157 \pm 163 for AAV-alb-GFP versus $3705 \pm 399$ for AAV-albRAMP2 mice), and this increase persisted for at least 4 months post-injection (Supplementary Figure $4 \mathrm{~A}-\mathrm{C}$ ). Lean mice treated with AAV-alb-RAMP2 had no readily apparent phenotypic differences, with comparable body weight to mice injected with a control AAV (AAV-albGFP) (Figure 4A). Although they exhibited a small reduction in glucose excursion during a glucose tolerance test following a 5-hour fast (Figure 4B), the same was not observed after a 24-hour fast (Figure 4C). They also exhibited no significant differences in glycaemic

Figure 3: Up-regulation of RAMP2 has spatiotemporal effects on intracellular signalling. A: cAMP dose response curves in Huh7-GCGR cells transfected with RAMP2 or control (pcDNA3.1) after 30 minutes stimulation with GCG, relative to forskolin $(10 \mu \mathrm{M})$ responses; $n=4$; 4-parameter fit of pooled data shown. B-E: Time-course of miniG $\alpha_{\mathrm{s}^{-}}$, miniG $\alpha_{q^{-}}$, miniG $\alpha_{\mathrm{i}^{-}}$and $\beta$-arrestin-2-LgBiT recruitment to GCGR-SmBiT following $100 \mathrm{nM}$ GCG stimulation measured by NanoBiT assays in the presence or absence of RAMP2, with AUC shown as insets. Data normalised to baseline (unstimulated) signal and expressed as Relative Light Units (RLU) as mean $\pm \mathrm{SEM}$ of $\mathrm{n}=4$ experiments; ${ }^{*} \mathrm{p}<0.05$. $\mathrm{F}$ : Ratio of AUCs in the presence vs. absence of RAMP2 for miniG $\alpha_{\mathrm{s}^{-}}$, miniG $\alpha_{q^{-}}$, miniG $\alpha_{j^{-}}$and $\beta$-arrestin-2-LgBiT recruitment to GCGR-SmBiT; data from B-E with each mini-G protein compared to $\beta$-arrestin-2 recruitment; ${ }^{*} \mathrm{p}<0.01$. G: Schematic of NanoBiT subcellular G protein activation assay: the GCGR and heterotrimeric $\mathrm{G}$ proteins are quiescent at the cell membrane. When glucagon binds to its receptor, recruited $\mathrm{G} \alpha_{\mathrm{S}}$ is activated and binds to Nb37 (1). Nb37-SmBiT complements CAAX-LgBiT upon plasma membrane activation. Following receptor internalisation (2), Nb37-SmBiT complements with Endofin-LgBiT indicating endosomal signalling. Created with BioRender.com. $\mathrm{H}$ and I: G $\alpha_{\mathrm{s}}$ activation at the plasma membrane and endosomal membranes, respectively; AUC over 30 minutes normalised to baseline; $n=6$; 4-parameter fit of pooled data shown. J: cAMP dose responses to GCG in control vs. WASH-out MEFs; $n=4$; 4-parameter fit of pooled data shown. K: MEF WASH-out cells co-transfected with SNAP-GCGR (labelled with SNAP-Surface 549, red) and Nb37-GFP (green) and stimulated with GCG for 30 minutes: examples of Nb37-GFP co-localised with internalised SNAP-GCGR indicating that it is actively signalling via G $\alpha_{\mathrm{S}}$ are arrowed; nuclei stained with DAPI (blue); scale bars $=10 \mu \mathrm{m}$. L: Western blot quantification of total SNAP-GCGR levels in HEK293T cells with and without RAMP2 co-expression following stimulation with $100 \mathrm{nM}$ GCG for 4 hours, normalised to tubulin as a loading control; data is mean \pm SEM of $n=3$ repeats (see Supplementary Figure $3 E$ for representative blots). M: HEK293T cells transfected with SNAP-GCGR (labelled with SNAP-Surface 649, blue) with or without CLIP-RAMP2 co-expression (labelled with CLIP-Surface 547 , red), and then treated with $100 \mathrm{nM} \mathrm{GCG}$ for $3 \mathrm{~h}$ before 5 min labelling with LysoTracker Green (green); scale bars = $10 \mu$ m; Manders' coefficient (SNAP-GCGR over lysotracker) quantified; $\mathrm{n}=5$; ***p $<0.001$. $\mathrm{N}$ : cAMP dose responses to GCG in Huh7-GCGR cells transfected with RAMP2 or control (pcDNA3.1) after a 24-hour stimulation period; $n=4$; 4-parameter fit of pooled data shown. Statistical significance analysed using paired t-test (panels B-E, $L$ and M) or one-way ANOVA with Sidak's post-hoc test ( $F$ ). 
Table 1 - Responses to glucagon stimulation in different cell types for the indicated times. Parameter estimates \pm SEM from responses depicted in Figure 3, $n=4-6, * p<0.05$, paired t-test.

\begin{tabular}{lll} 
& \multicolumn{2}{l}{ Huh7-GCGR acute (30 minutes) cAMP } \\
\cline { 2 - 3 } & -RAMP2 & +RAMP2 \\
\hline Emax & $141.2 \pm 8.6$ & $201.6 \pm 9.6^{*}$ \\
LogEC50 & $-10.40 \pm 0.14$ & $-10.21 \pm 0.20$ \\
\hline & MEF flox/flox cAMP & MEF WASH-out cAMP \\
\hline Emax & $8.49 \pm 1.14$ & $18.3 \pm 2.8^{*}$ \\
LogEC50 & $-7.33 \pm 0.25$ & $-7.17 \pm 0.31$ \\
\hline & HEK293T plasma membrane GCGR activity (NanoBiT) \\
\hline & -RAMP2 & + RAMP2 \\
\hline Emax & $3.01 \pm 0.26$ & $2.48 \pm 0.32$ \\
LogEC50 & $-8.05 \pm 0.06$ & $-7.81 \pm 0.15$ \\
\hline & HEK293T endosomal membrane GCGR activity (NanoBiT) \\
\hline & -RAMP2 & + RAMP2 \\
\hline Emax & $0.49 \pm 0.15$ & $0.92 \pm 0.08^{*}$ \\
LogEC50 & $-7.50 \pm 0.35$ & $-8.24 \pm 0.24^{*}$ \\
\hline & Huh7-GCGR prolonged (24 hours) CAMP \\
\hline & -RAMP2 & + RAMP2 \\
\hline Emax & $34.6 \pm 5.4$ & $16.4 \pm 3.2^{*}$ \\
LogEC50 & $-7.87 \pm 0.26$ & $-7.59 \pm 0.16$ \\
\hline & Primary hepatocytes cAMP \\
\hline & -RAMP2 & + RAMP2 \\
\hline Emax & 458 \pm 146 & $521 \pm 84$ \\
LogEC50 & $-10.0 \pm 0.81$ & $-10.5 \pm 0.49$ \\
\hline &
\end{tabular}

responses when subjected to a GCG challenge or insulin tolerance test (Figure 4D,E). As compensatory mechanisms could mask differences in GCG signalling between the two cohorts, we harvested primary hepatocytes and measured cAMP in response to GCG: although there was a trend for higher cAMP efficacy and potency in AAV-alb-RAMP2 hepatocytes, this trend was not statistically significant (Figure $4 \mathrm{~F}$ and Table 1).

The same cohort of mice were next transferred to a high-fat diet, and metabolic tests were performed when the mice were obese; again, there were no apparent differences between the groups (Supplementary Figure 4D-H). Given that the up-regulated expression of RAMP2 was variable between mice, we performed a correlation analysis between hepatic RAMP2 levels and AUC following a GCG challenge test in obese mice, but did not observe any correlation (Supplementary Figure 4I). In this experimental setting, therefore, upregulation of hepatic RAMP2 did not have a dramatic effect on carbohydrate metabolism in adult male mice.

\section{DISCUSSION}

Here, we demonstrate that RAMP2 modulates both trafficking and function of the GCGR. In both HEK293T and Huh7 cells, RAMP2 promotes intracellular accumulation of the GCGR, both basally and following agonist stimulation. In agreement with recent work [38], our data indicate that, in the absence of RAMP2, the GCGR internalises, deposits its ligand intracellularly and subsequently recycles to the plasma membrane, a process that is disrupted by blocking recycling by either using monensin or using cells where the WASH complex is knocked out. Given that FITC-GCG rapidly accumulates intracellularly in both the presence and absence of RAMP2 (where the GCGR is primarily detected at the plasma membrane), the effect of RAMP2 on GCGR subcellular location is likely due to a reduction in the rate of GCGR recycling. RAMPs have previously been shown to alter the recycling rate of GPCRs: Bomberger et al. demonstrated that RAMP3 is responsible for retaining the adrenomedullin receptor 2 (RAMP3/CRLR) intracellularly after agonist stimulation and internalisation [32]. More recently, Mackie et al. showed that RAMP3 is required for rapid recycling of the atypical chemokine receptor ACKR3 [33]. Taken together, these studies appear to indicate that RAMPs have differential effects on regulating receptor recycling rates, depending on the GPCR in question. We speculated that endosomal GCGR would be more likely to be targeted for degradation over prolonged agonist incubation times. We indeed observed increased lysosomal localisation as well as a trend towards increased degradation of GCGR in the presence of RAMP2 after longer term GCG exposure, a finding previously made for RAMP3 and the CRLR [32]. Given that RAMPs are ubiquitously present in many tissue types, modulation of their expression levels may play important roles in the control of physiological receptor turnover via fine tuning of the balance between receptor recycling and degradation rates.

The interaction of RAMP2 with GCGR has functional consequences: here, we demonstrate that up-regulation of RAMP2 acutely increases agonist-stimulated cAMP production in hepatoma cells, a phenomenon that has previously been shown in other non-hepatocyte cell lines $[19,20]$. We also observe a similar increase in cAMP production when GCGR is artificially forced to accumulate intracellularly with blocked recycling in WASH-out MEFs, indicating that intracellular GCGR sequestration triggered by RAMP2 co-expression is likely responsible for the detected increase in accumulated cAMP. Indeed, using a novel NanoBiT subcellular $G$ protein activation assay we were able to demonstrate that, in the presence of RAMP2, there is greater GCGR activity from endosomes specifically. Data from other secretin-family GPCRs, including the parathyroid hormone receptor and the GLP-1R, which both signal via $\mathrm{G} \alpha_{\mathrm{S}}$ from early endosomes [39-41], indicates that the spatiotemporal regulation of signalling is paramount to modulating receptor outputs, with intracellular signalling usually associated with more sustained responses [42,43]. Our data suggests that a similar phenomenon may be true for the GCGR/RAMP2 complex, a possibility that deserves further investigation, especially given its important ramifications for therapeutic targeting of the receptor [44]. We also observed a RAMP2-induced bias away from $\beta$-arrestin-2 recruitment at the GCGR, which corroborates our previous findings in $\mathrm{CHO}$ cells using a beta-galactosidase fragment complementation assay [19]. $\beta$-arrestin recruitment has potentially variable effects on $G$ protein-dependent GPCR signalling: it may terminate G proteinmediated signalling via uncoupling of $G$ proteins and increased activity of cAMP phosphodiesterases [45,46], but it has also been hypothesised to facilitate sustained endosomal signalling by the formation of GPCR-G protein- $\beta$-arrestin supercomplexes [44]. We have previously demonstrated that, for the GCGR and related GPCRs, absence of $\beta$-arrestins increases overall agonist-stimulated cAMP production [25]. In the present study, bias away from $\beta$-arrestin-2 recruitment could therefore explain the overall increase in glucagonstimulated cAMP accumulation when RAMP2 is over-expressed. Because $\beta$-arrestins have been shown to be involved in the recycling of GCGR from intracellular compartments back to the plasma membrane, a reduction in their recruitment could be responsible for the increased intracellular retention of GCGR in the presence of RAMP2 [47]. Signalling bias conferred by RAMP2 on the GCGR has previously been reported in a chimeric yeast system, where RAMP2 appeared to reduce recruitment of $\mathrm{G} \alpha_{\mathrm{i}}$ and increase recruitment of $\mathrm{G} \alpha_{\mathrm{s}}$ to the 
A
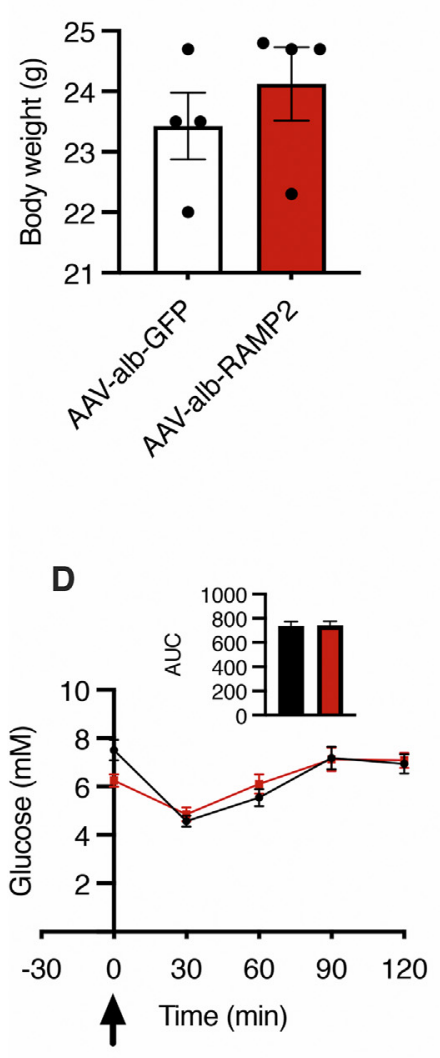

Insulin
B

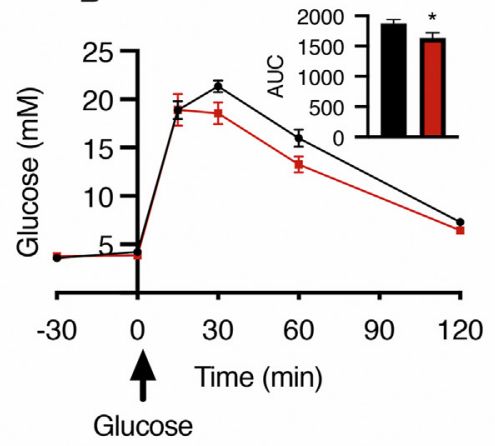

E

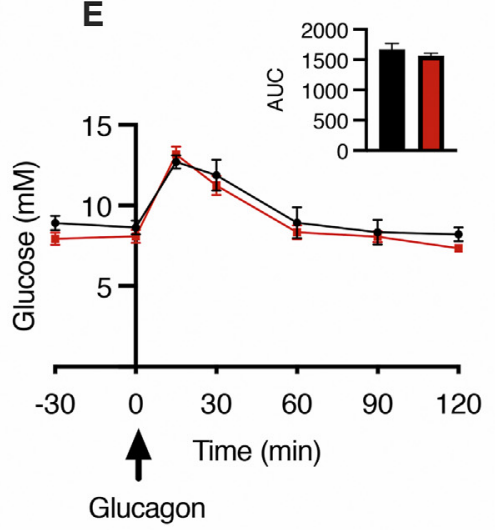

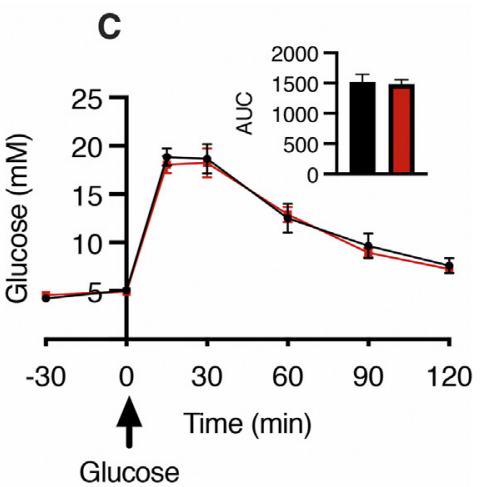

F

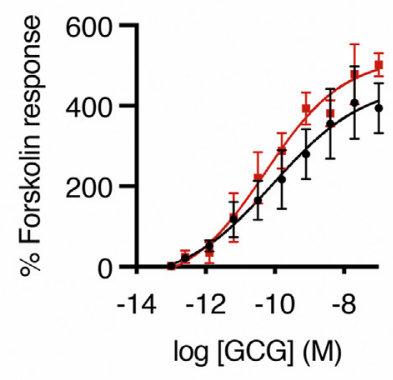

$\rightarrow$ AAV-alb-GFP

$\rightarrow \quad$ AAV-alb-RAMP2

Figure 4: Up-regulation of hepatic RAMP2 in lean adult male mice is not associated with a phenotypic change. A: Mouse body weight after 18 days upregulation of hepatic mouse RAMP2 (AAV-alb-RAMP2) or control (AAV-alb-GFP); $n=4$; data are mean \pm SEM. B: Glucose tolerance test after 5-hour fast. C: Glucose tolerance test after 24-hour fast. D: Insulin tolerance test. E: Glucagon challenge. B-E experiments performed in mice 3-4 weeks post-AAV injection; $n=9-10$ per group; data are mean \pm SEM, with AUC shown as inset; unpaired t-test; ${ }^{*} \mathrm{p}<0.05$. F: cAMP dose response to GCG in isolated primary hepatocytes from AAV-alb-GFP and AAV-alb-RAMP2 mice, normalised to forskolin (10 $\mu$ M) responses; $n=4$; 4-parameter fit of pooled data shown; $n=7$ separate mice from each cohort harvested over 4 days.

receptor [20]; however, we observed no differences in recruitment to mini-G $\alpha_{s},-G \alpha_{i}$ or $-G \alpha_{q}$, albeit with very low overall levels of $\mathrm{G} \alpha_{\mathrm{i}}$ recruitment, in our experimental system.

There is very little data in the literature investigating the effects of RAMP upregulation on GPCR activity in vivo. Our in vitro findings suggested that RAMP2 may play a role in modifying GCGR activity in hepatocytes, therefore potentially impacting the processes of glycogenolysis, gluconeogenesis and fatty acid oxidation. Here, however, aside from a small improvement in glucose tolerance after a 5-hour fast and a trend to greater efficacy for agonist-stimulated cAMP production in isolated hepatocytes, we did not find major effects on carbohydrate metabolism from over-expressing hepatic RAMP2 in either lean or obese mice. It remains to be investigated whether hepatic down-regulation (rather than overexpression) of RAMP2, or RAMP2 up-regulation in other nutritional contexts may yield a biological effect on glucagon signalling in vivo. The level of up-regulation achieved in our system (3-fold) may also not have been sufficient to bring out a phenotype; however, it is worth noting that this level is comparable to the increase in hepatic RAMP2 observed in a rodent model of cirrhosis [48]. There may also be species-specificity of RAMP2 effects; the in vitro data here concerns human RAMP2 interacting with human GCGR, whereas in our in vivo experiment we manipulated hepatic murine RAMP2 and studied effects of its interaction with murine GCGR. Furthermore, we cannot rule out an effect of RAMP2 modulation of GCGR in other pathophysiological contexts [48] or in different target organs.

\section{CONCLUSION}

In the absence of RAMP2, GCGR is found predominantly at the cell surface at a steady state, but this localisation is underlaid by continuous cycles of receptor internalisation and recycling following acute ligand stimulation. In the presence of RAMP2, GCGR accumulates intracellularly, both under basal conditions and following GCG stimulation. Upon acute stimulation with GCG, RAMP2 co-expression results in a short-term increase in cAMP accumulation, which may be explained by more efficient signalling from GCGRs retained in endosomes. RAMP2 is also associated with a bias away from $\beta$-arrestin-2 recruitment, which may provide a mechanism for the retention of GCGR intracellularly. Over prolonged periods of GCG stimulation, there is a trend towards increased GCGR degradation, potentially associated with a long-term reduction in efficacy for cAMP production in the presence of RAMP2. Finally, we present in vivo data suggesting that 
these effects on signalling do not lead to a readily observable phenotype in lean and obese mice following upregulation of hepatic murine RAMP2. Further work is needed to determine the circumstances in which RAMP2 might play a role in regulating GCGR trafficking and signalling in vivo in the liver.

\section{ACKNOWLEDGEMENTS}

We thank David MacDonald for performing the mouse tail vein injections, Eleni Vloumidi for primary hepatocyte harvest and Roger K. Sunahara at University of California, San Diego for helpful discussion regarding the development of the NanoBiT-Nb37 assay. We acknowledge the Facility for Imaging by Light Microscopy (FILM) at Imperial College London, which is partly supported by funding from the Wellcome Trust (grant 104931/Z/14/Z) and BBSRC (grant BB/L015129/1), and the particular help of Stephen Rothery in macro design for quantification. ERM was supported by a Royal College of Surgeons of England one-year research fellowship and an MRC Clinical Research Training Fellowship while working on this project. AT and DC are funded by the MRC (MR/R010676/1 and MC-A654-5QB10, respectively). AT also acknowledges support from Diabetes UK and the European Federation for the Study of Diabetes. BJ is supported by the MRC, European Federation for the Study of Diabetes, Society for Endocrinology, British Society for Neuroendocrinology, and the NIHR Imperial Biomedical Research Centre. TT and SRB are funded by the NIHR Imperial BRC. Al was funded by the LEAP JP20gm0010004 and the BINDS JP20am0101095 from the Japan Agency for Medical Research and Development (AMED), JST Moonshot Research and Development Program JPMJMS2023 from Japan Science and Technology Agency (JST), and The Uehara Memorial Foundation. The Department of Metabolism, Digestion and Reproduction at Imperial College London, UK, is funded by grants from the MRC and the Biotechnology and Biological Sciences Research Council and is supported by the NIHR Imperial Biomedical Research Centre (BRC) Funding Scheme. The views expressed are those of the authors and not necessarily those of the abovementioned funders, the NHS, the NIHR, or the Department of Health.

\section{CONFLICT OF INTEREST}

None declared.

\section{APPENDIX A. SUPPLEMENTARY DATA}

Supplementary data to this article can be found online at https://doi.org/10.1016/j. molmet.2021.101296.

\section{REFERENCES}

[1] Mayo, K.E., Miller, L.J., Bataille, D., Dalle, S., Göke, B., Thorens, B., et al., 2003. International Union of Pharmacology. XXXV. The glucagon receptor family. Pharmacological Reviews 55(1):167-194.

[2] Marroqui, L., Alonso-Magdalena, P., Merino, B., Fuentes, E., Nadal, A., Quesada, I., 2014. Nutrient regulation of glucagon secretion: involvement in metabolism and diabetes. Nutrition Research Reviews 27(1):48-62.

[3] Penhos, J.C., Wu, C.H., Daunas, J., Reitman, M., Levine, R., 1966. Effect of glucagon on the metabolism of lipids and on urea formation by the perfused rat liver. Diabetes 15(10):740-748.

[4] Nafikov, R.A., Ametaj, B.N., Bobe, G., Koehler, K.J., Young, J.W., Beitz, D.C., 2006. Prevention of fatty liver in transition dairy cows by subcutaneous injections of glucagon. Journal of Dairy Science 89(5):1533-1545.

[5] Demant, M., Bagger, J.I., Suppli, M.P., Lund, A., Gyldenlove, M., Hansen, K.B., et al., 2018. Determinants of fasting hyperglucagonemia in patients with type 2 diabetes and nondiabetic control subjects. Metabolic Syndrome and Related Disorders 16(10):530-536.
[6] Knop, F.K., Vilsbøøl, T., Madsbad, S., Holst, J.J., Krarup, T., 2007. Inappropriate suppression of glucagon during OGTT but not during isoglycaemic i.v. glucose infusion contributes to the reduced incretin effect in type 2 diabetes mellitus. Diabetologia 50(4):797-805.

[7] Sammons, M.F., Lee, E.C.Y., 2015. Recent progress in the development of small-molecule glucagon receptor antagonists. Bioorganic \& Medicinal Chemistry Letters 25(19):4057-4064.

[8] Sánchez-Garrido, M.A., Brandt, S.J., Clemmensen, C., Müller, T.D., DiMarchi, R.D., Tschöp, M.H., 2017. GLP-1/glucagon receptor co-agonism for treatment of obesity. Diabetologia 60(10):1851-1861.

[9] Buenaventura, T., Kanda, N., Douzenis, P.C., Jones, B., Bloom, S.R., Chabosseau, P., et al., 2018. A targeted RNAi screen identifies endocytic trafficking factors that control GLP-1 receptor signaling in pancreatic $\beta$-cells. Diabetes 67(3):385-399.

[10] Fang, Z., Chen, S., Pickford, P., Broichhagen, J., Hodson, D.J., Corrêa, I.R., et al., 2020. The influence of peptide context on signaling and trafficking of glucagon-like peptide-1 receptor biased agonists. ACS Pharmacology Translational Science 3(2):345-360.

[11] Jones, B., Buenaventura, T., Kanda, N., Chabosseau, P., Owen, B.M., Scott, R. et al., 2018. Targeting GLP-1 receptor trafficking to improve agonist efficacy. Nature Communications 9(1):1602-1617.

[12] Roed, S.N., Nohr, A.C., Wismann, P., Iversen, H., Brauner-Osborne, H., Knudsen, S.M., et al., 2015. Functional consequences of glucagon-like peptide-1 receptor cross-talk and trafficking. Journal of Biological Chemistry 290(2):1233-1243.

[13] Abdullah, N., Beg, M., Soares, D., Dittman, J.S., McGraw, T.E., 2016. Downregulation of a GPCR by $\beta$-arrestin2-mediated switch from an endosomal to a TGN recycling pathway. Cell Reports 17(11):2966-2978.

[14] Barbash, S., Lorenzen, E., Persson, T., Huber, T., Sakmar, T.P., 2017. GPCRs globally coevolved with receptor activity-modifying proteins, RAMPs. Proceedings of the National Academy of Sciences of the United States of America 114(45):12015-12020.

[15] Lorenzen, E., Dodig-Crnkovic, T., Kotliar, I.B., Pin, E., Ceraudo, E., Vaughan, R.D., et al., 2019. Multiplexed analysis of the secretin-like GPCRRAMP interactome. Science Advances 5(9):eaaw2778.

[16] Klein, K.R., Matson, B.C., Caron, K.M., 2016. The expanding repertoire of receptor activity modifying protein (RAMP) function. Critical Reviews in Biochemistry and Molecular Biology 51(1):65-71.

[17] Tilakaratne, N., Christopoulos, G., Zumpe, E.T., Foord, S.M., Sexton, P.M., 2000. Amylin receptor phenotypes derived from human calcitonin receptor/ RAMP coexpression exhibit pharmacological differences dependent on receptor isoform and host cell environment. Journal of Pharmacology and Experimental Therapeutics 294(1):61-72.

[18] Christopoulos, A., Christopoulos, G., Morfis, M., Udawela, M., Laburthe, M., Couvineau, A., et al., 2003. Novel receptor partners and function of receptor activity-modifying proteins. Journal of Biological Chemistry 278(5):3293-3297.

[19] Cegla, J., Jones, B.J., Gardiner, J.V., Hodson, D.J., Marjot, T., McGlone, E.R., et al., 2017. RAMP2 influences glucagon receptor pharmacology via trafficking and signaling. Endocrinology 158(8):2680-2693.

[20] Weston, C., Lu, J., Li, N., Barkan, K., Richards, G.O., Roberts, D.J., et al., 2015. Modulation of glucagon receptor pharmacology by receptor activitymodifying protein-2 (RAMP2). Journal of Biological Chemistry 290(38): 23009-23022.

[21] Jones, B.J., Scopelliti, R., Tomas, A., Bloom, S.R., Hodson, D.J., Broichhagen, J., 2017. Potent prearranged positive allosteric modulators of the glucagon-like peptide-1 receptor. Chemistry 0pen 6(4):501-505.

[22] Owji, A.A., Smith, D.M., Coppock, H.A., Morgan, D.G., Bhogal, R., Ghatei, M.A., et al., 1995. An abundant and specific binding site for the novel vasodilator adrenomedullin in the rat. Endocrinology 136(5):2127-2134.

[23] Woods, A., Heslegrave, A.J., Muckett, P.J., Levene, A.P., Clements, M., Mobberley, M., et al., 2011. LKB1 is required for hepatic bile acid transport 
and canalicular membrane integrity in mice. Biochemical Journal 434(1): $49-60$.

[24] Aaron, J.S., Taylor, A.B., Chew, T.-L., 2018. Image co-localization - cooccurrence versus correlation. Journal of Cell Science 131(3):jcs211847.

[25] Jones, B., McGlone, E.R., Fang, Z., Pickford, P., Correa Jr., I.R., Oishi, A., et al., 2021. Genetic and biased agonist-mediated reductions in beta-arrestin recruitment prolong CAMP signalling at glucagon family receptors. Journal of Biological Chemistry 296:100133.

[26] Zhu, L., Rossi, M., Cui, Y., Lee, R.J., Sakamoto, W., Perry, N.A., et al., 2017. Hepatic beta-arrestin 2 is essential for maintaining euglycemia. Journal of Clinical Investigation 127(8):2941-2945.

[27] Gomez, T.S., Gorman, J.A., de Narvajas, A.A.-M., Koenig, A.O., Billadeau, D.D., 2012. Trafficking defects in WASH-knockout fibroblasts originate from collapsed endosomal and lysosomal networks. Molecular Biology of the Cell 23(16):3215-3228.

[28] King, J.S., Gueho, A., Hagedorn, M., Gopaldass, N., Leuba, F., Soldati, T., et al., 2013. WASH is required for lysosomal recycling and efficient autophagic and phagocytic digestion. Molecular Biology of the Cell 24(17): $2714-2726$.

[29] Buckley, C.M., Gopaldass, N., Bosmani, C., Johnston, S.A., Soldati, T., Insall, R.H., et al., 2016. WASH drives early recycling from macropinosomes and phagosomes to maintain surface phagocytic receptors. Proceedings of the National Academy of Sciences of the United States of America 113(40): E5906-E5915.

[30] Bahouth, S.W., Nooh, M.M., 2017. Barcoding of GPCR trafficking and signaling through the various trafficking roadmaps by compartmentalized signaling networks. Cellular Signalling 36:42-55.

[31] Stein, B.S., Bensch, K.G., Sussman, H.H., 1984. Complete inhibition of transferrin recycling by monensin in K562 cells. Journal of Biological Chemistry 259(23): 14762-14772.

[32] Bomberger, J.M., Spielman, W.S., Hall, C.S., Weinman, E.J., Parameswaran, N., 2005. Receptor activity-modifying protein (RAMP) isoformspecific regulation of adrenomedullin receptor trafficking by NHERF-1. Journal of Biological Chemistry 280(25):23926-23935.

[33] Mackie, D.I., Nielsen, N.R., Harris, M., Singh, S., Davis, R.B., Dy, D., et al., 2019. RAMP3 determines rapid recycling of atypical chemokine receptor-3 for guided angiogenesis. Proceedings of the National Academy of Sciences of the United States of America 116(48):24093-24099.

[34] Wan, Q., Okashah, N., Inoue, A., Nehmé, R., Carpenter, B., Tate, C.G., et al., 2018. Mini G protein probes for active G protein-coupled receptors (GPCRs) in live cells. Journal of Biological Chemistry 293(19):7466-7473.

[35] Irannejad, R., Tomshine, J.C., Tomshine, J.R., Chevalier, M., Mahoney, J.P., Steyaert, J., et al., 2013. Conformational biosensors reveal GPCR signalling from endosomes. Nature 495(7442):534-538.
[36] Lucey, M., Ashik, T., Marzook, A., Wang, Y., Goulding, J., Oishi, A., et al., 2021. Acylation of the incretin peptide exendin-4 directly impacts GLP-1 receptor signalling and trafficking. Molecular Pharmacology 100(1):000270.

[37] Cullen, P.J., Steinberg, F., 2018. To degrade or not to degrade: mechanisms and significance of endocytic recycling. Nature reviews. Molecular Cell Biology 19(11):679-696.

[38] Kaur, S., Chen, Y., Shenoy, S.K., 2020. Agonist-activated glucagon receptors are deubiquitinated at early endosomes by two distinct deubiquitinases to facilitate Rab4a-dependent recycling. Journal of Biological Chemistry 295(49): 16630-16642.

[39] Ferrandon, S., Feinstein, T.N., Castro, M., Wang, B., Bouley, R., Potts, J.T., et al., 2009. Sustained cyclic AMP production by parathyroid hormone receptor endocytosis. Nature Chemical Biology 5(10):734-742.

[40] Manchanda, Y., Bitsi, S., Kang, Y., Jones, B., Tomas, A., 2021. Spatiotemporal control of GLP-1 receptor activity. Current Opinion in Endocrine and Metabolic Research 16:19-27.

[41] Girada, S.B., Kuna, R.S., Bele, S., Zhu, Z., Chakravarthi, N.R., DiMarchi, R.D., et al., 2017. Gas regulates Glucagon-Like Peptide 1 Receptor-mediated cyclic AMP generation at Rab5 endosomal compartment. Molecular Metabolism 6(10):1173-1185.

[42] Plouffe, B., Thomsen, A.R.B., Irannejad, R., 2020. Emerging role of compartmentalized G protein-coupled receptor signaling in the cardiovascular field. ACS Pharmacology Translational Science 3(2):221-236.

[43] Pavlos, N.J., Friedman, P.A., 2017. GPCR signaling and trafficking: the long and short of it. Trends in Endocrinology and Metabolism: Trends in Endocrinology and Metabolism 28(3):213-226.

[44] Thomsen, A.R.B., Jensen, D.D., Hicks, G.A., Bunnett, N.W., 2018. Therapeutic targeting of endosomal G-protein-coupled receptors. Trends in Pharmacological Sciences 39(10):879-891.

[45] Cahill 3rd, T.J., Thomsen, A.R., Tarrasch, J.T., Plouffe, B., Nguyen, A.H., Yang, F., et al., 2017. Distinct conformations of GPCR-beta-arrestin complexes mediate desensitization, signaling, and endocytosis. Proceedings of the National Academy of Sciences of the United States of America 114(10): 2562-2567.

[46] Reiter, E., Ayoub, M.A., Pellissier, L.P., Landomiel, F., Musnier, A., Trefier, A., et al., 2017. beta-arrestin signalling and bias in hormone-responsive GPCRs. Molecular and Cellular Endocrinology 449:28-41.

[47] Krilov, L., Nguyen, A., Miyazaki, T., Unson, C.G., Bouscarel, B., 2008. Glucagon receptor recycling: role of carboxyl terminus, beta-arrestins, and cytoskeleton. American Journal of Physiology - Cell Physiology 295(5):C1230-C1237.

[48] Hwang, I.S.S., Tang, F., Leung, P.P., Li, Y.Y., Fan, S.T., Luk, J.M.C., 2006. The gene expression of adrenomedullin, calcitonin-receptor-like receptor and receptor activity modifying proteins (RAMPs) in CCI4-induced rat liver cirrhosis. Regulatory Peptides 135(1-2):69-77. 